\title{
Bilateral Parapharyngeal Abscess Complicated with Trismus, Airway Obstruction and Bleeding
}

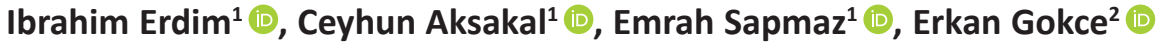 \\ ${ }^{1}$ Tokat Gaziosmanpasa University, Health Education and Training Hospital, Department of Otorhinolaryngology, Tokat, Turkiye \\ ${ }^{2}$ Tokat Gaziosmanpasa University, Health Education and Training Hospital, Department of Radiology, Tokat, Turkiye
}

ORCID ID: I.E. 0000-0003-1840-6052; C.A. 0000-0001-9770-1513; E.S. 0000-0002-0346-8704; E.G. 0000-0003-3947-2972

Citation: Erdim I, Aksakal C, Sapmaz E, Gokce E. Bilateral parapharyngeal abscess complicated with trismus, airway obstruction and bleeding. Tr-ENT. Published online March 2, 2022. https://doi.org/10.26650/Tr-ENT.2021.1015708

\section{ABSTRACT}

Bilateral parapharyngeal abscess is a very rare condition. Placing pressure on the airway on both sides increases the risk of respiratory arrest. Intubation cannot be performed due to the trismus caused by the infection in the mastication muscles, and the need to create an emergency airway can only be provided by invasive means. The adjacency of the region to major vessels and their branches can lead to massive hemorrhages since the infection erodes the vascular wall. In a case of bilateral parapharyngeal abscesses which resulted in respiratory arrest due to the trismus, we were able to ensure airway safety using a coniotomy and subsequent tracheotomy. The presence of massive hemorrhages on both sides in the early postoperative period caused us to remain constantly vigilant until the full recovery of the patient in terms of clinical, laboratory and radiological parameters.

Keywords: Bilateral, parapharyngeal, abscess, airway, bleeding

\section{INTRODUCTION}

Because of easy access to antibiotics and physicians, the incidence of parapharyngeal abscesses has been on decrease. However, it is still a condition that should be kept in mind and not be disregarded $(1,2)$. The most common etiological causes of parapharyngeal abscesses are dental infections and tonsillitis $(1,3,4)$. Mediastinitis can cause serious complications such as internal jugular vein thrombosis, carotid artery rupture, cavernous sinus thrombosis, pericarditis, sepsis, epidural abscess and even mortality $(1-3,5)$.

In our literature review, we found only one case in which a bilateral parapharyngeal abscess was presented (6). In that case report, the bilateral parapharyngeal abscess was complicated with sepsis and pulmonary candidiasis (6). In the present report, we introduce a bilateral parapharyngeal abscess case which was complicated with trismus, upper respiratory obstruction and bleeding.

\section{CASE REPORT}

A 28-year-old male patient was admitted to our clinic with the complaints of trismus, difficulty swallowing, neck pain and fever. Our examination showed widespread edema and sensitivity in the neck. An oropharyngeal examination could not be performed since he had trismus. In addition, a laryngeal examination could not be performed due to his inability to tolerate the flexible endoscopic examination. Nasal cavity and nasopharyngeal examinations were normal. The patient's temperature was $38.9^{\circ} \mathrm{C}$, leukocytes number was $22830 / \mathrm{ml}$, neutrophil count was $20150 / \mathrm{ml}$, and C-reactive protein level was $287.51 \mathrm{mg} / \mathrm{l}$.

A contrast-enhanced neck CT scan was requested to detect the patient's infection focus. Upon the onset of respiratory distress, which began about one hour after the CT scan, the patient was taken to the operating room for emergency airway intervention. Due to the development of respiratory arrest, an emergency coniotomy was performed. Immediately afterwards, a tracheotomy was carried out and respiratory

Corresponding Author: Ibrahim Erdim E-mail: ibrahim_erdim@hotmail.com

Submitted: 27.10.2021 • Revision Requested: 24.01.2022 • Last Revision Received: 01.02.2022 • Accepted: 17.02.2022 • Published Online: 02.03 .2022 
safety was ensured. Bilateral parapharyngeal, sublingual and submandibular abscess foci detected in the CT scan (Figure $1 \mathrm{a}, \mathrm{b}$, Figure $2 \mathrm{a}, \mathrm{b}, \mathrm{c}$ ) were drained by bilateral submandibular incision. The submandibular gland posterolateral was retracted, dissection was continued upwards, and the parapharyngeal abscess foci were reached. By applying finger dissection, all loculations were combined to completely drain the abscess. After the control of bleeding, Penrose drainage was placed in both parapharyngeal spaces, and the operation was completed. Interventions were performed again under general anesthesia since the patient had bleeding on the left side on the postoperative first day and the right side on the postoperative second day. The patient's hemoglobin value during the admission was $13.76 \mathrm{~g} / \mathrm{dl}$. However, after the second bleeding, the hemoglobin level fell to $7.55 \mathrm{~g} / \mathrm{dl}$, and therefore, two units of erythrocyte suspension transfusion were applied.

Intravenous antibiotic therapy was applied after his first admission using ceftriaxone $2 \times 2 \mathrm{~g} / 100 \mathrm{ml}$ and metronidazole $3 \times 500 \mathrm{mg} / 100 \mathrm{ml}$. Gram positive cocci were observed in the microscopic examination of the stained abscess fluid taken during surgical drainage. Non-hemolytic streptococcus growth was observed in the culture. The current treatment was continued when it was revealed that the factor was sensitive to the antibiotic therapy which was started empirically.

The patient's oral opening reached a sufficient level on the $18^{\text {th }}$ day of treatment. After the trismus was relaxed and the airway opening was ensured, his tracheotomy was closed, the nasogastric catheter was removed, and oral nutrition was started. The oropharyngeal examination, which could be performed after the oral opening was achieved, showed a caries on the right third molar tooth. Upon consultation with dentistry, the decayed tooth was extracted. After determining that laboratory examinations and contrast-enhanced neck tomography were normal on the $22^{\text {nd }}$ day of the patient's admission (Figure 1c, Figure 2d), the patient was discharged with recovery. No pathology was detected during the patient's six-month follow-ups.

\section{DISCUSSION}

The frequency of parapharyngeal abscesses has decreased with the widespread use of antibiotics. However, this decreased frequency brings the danger of neglecting this condition. In addition, unlike other deep neck infections, parapharyngeal abscesses could be difficult to identify by inspection or physical examination. Here, contrast-enhanced tomography comes to the fore. Contrast-enhanced neck tomography is very useful in detecting the infection focus in symptoms such as dysphagia, odynophagia, fever, neck pain, swelling and pain in the neck, and elevated laboratory parameters indicating infection $(2,3,5)$.

Different clinical treatment modalities are available for the treatment of parapharyngeal abscesses. The approach may vary depending on the general condition of the patient, and the size, location and complications of the abscess. There are studies indicating that localized, small-sized abscesses without complications should be treated only with intravenous antibiotic therapy (2). However, this requires close follow-up of patients (2). In cases where the parapharyngeal abscess is small in size, needle aspiration along with parenteral antibiotic therapy is another treatment alternative (2). Abscess drainage through surgical incision, on the other hand, can be carried out through external skin incision as well as through internal pharyngeal mucosa incision $(1,2,4)$. In cases where parapharyngeal abscess originates from peritonsillar abscess, a tonsillectomy could be included in the treatment and the abscess is drained from this space (2). However, in this case, it is important not to ignore the risk of bleeding brought on by hot tonsillectomy. With the advances in the medical technologies, it is possible to reach the uniloculated parapharyngeal abscess and achieve drainage with the help of an endoscope from the nasopharyngeal (7) or oropharyngeal region. However, in a.

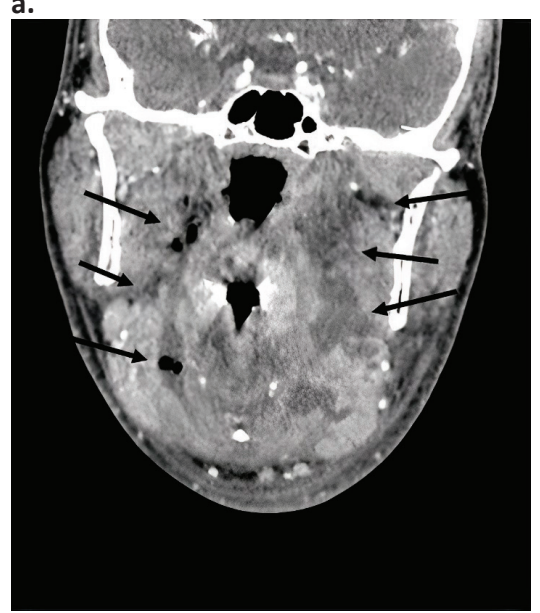

b.

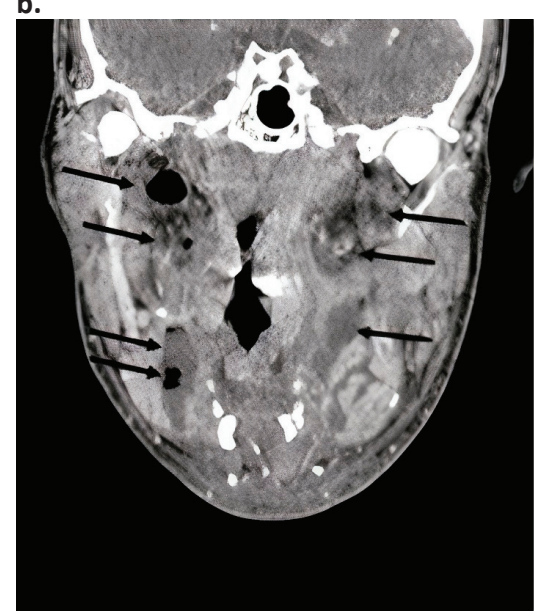

c.

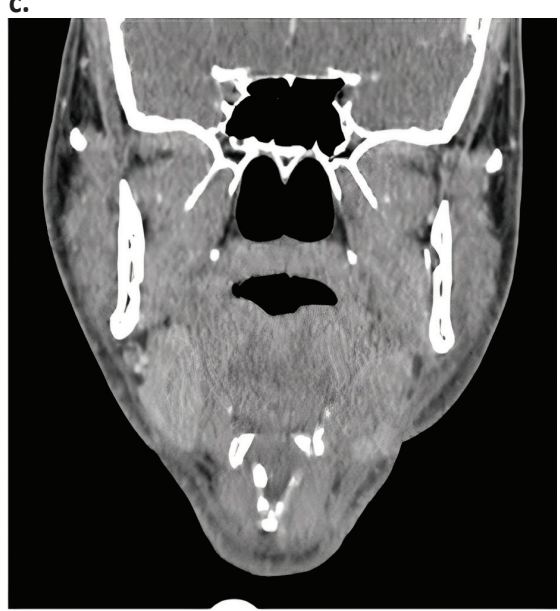

Figure 1: a) and b) Air inside the abscess foci (arrows) extending from the bilateral parapharyngeal distances to the submandibular and sublingual distances are shown in the reformatted, contrast-enhanced computed tomography (CT) images on the coronal plane. c) Completely regressed abscesses are shown in the reformatted, contrast-enhanced CT images on the coronal plane after the treatment. 
a.

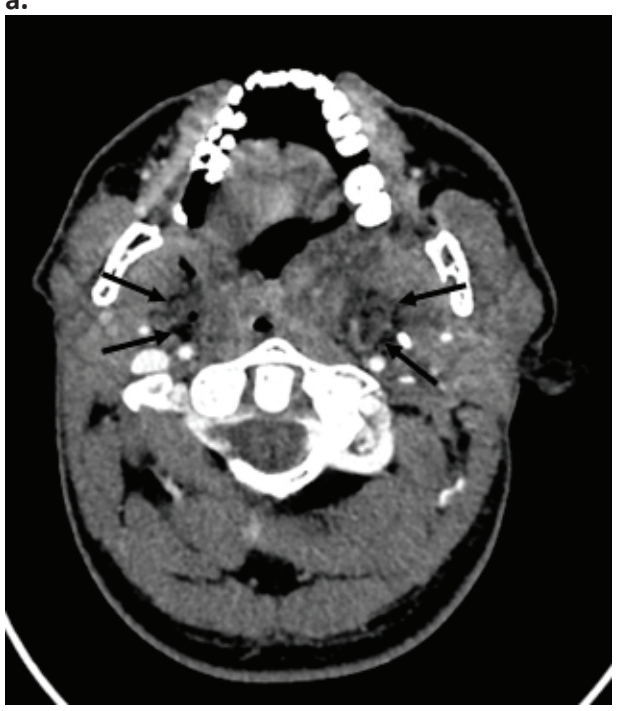

b.

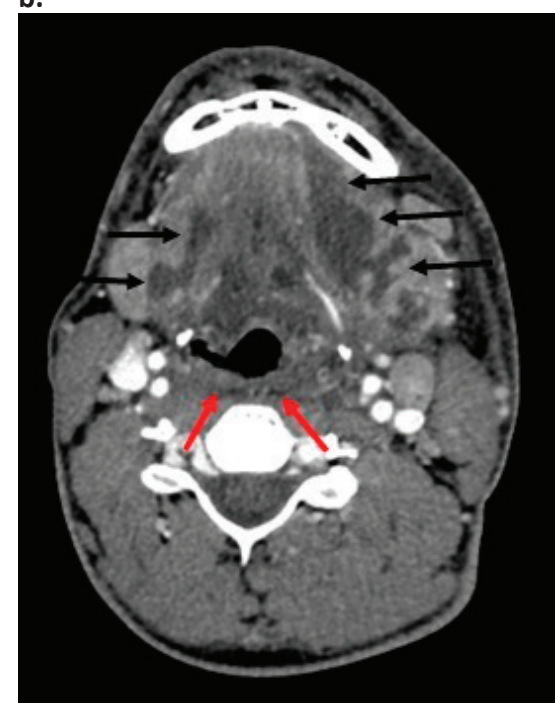

c.

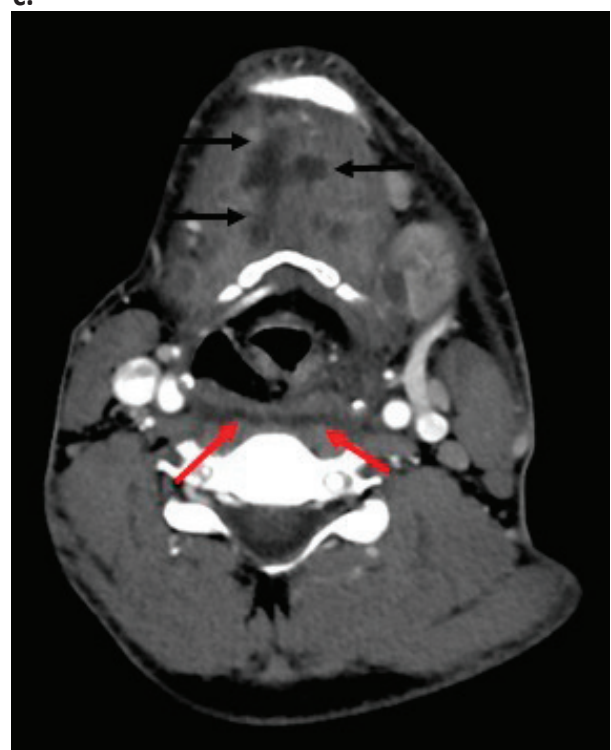

d.

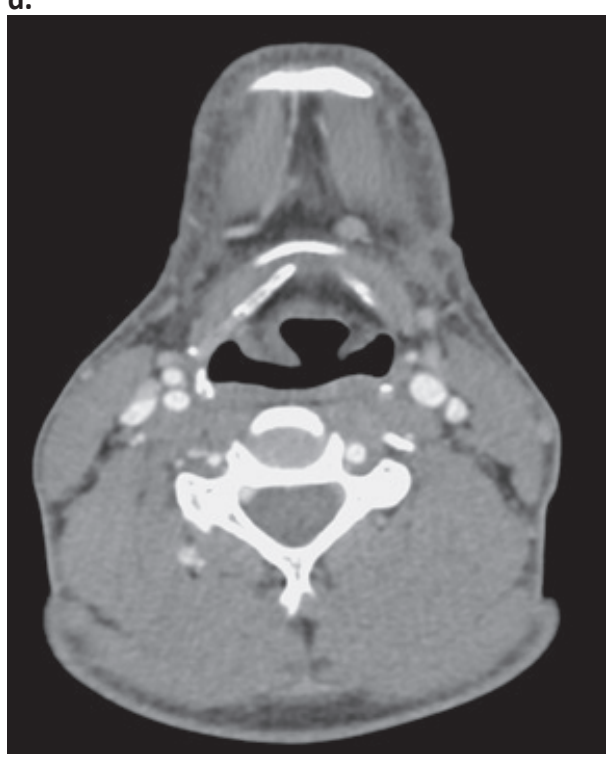

Figure 2: a) Axial CT images show a fluid collection extending from the bilateral submandibular area to the parapharyngeal distance (black arrows) including air densities. b) and c) Although mild edema is observed in the retropharyngeal space (red arrows), no abscess is evident. Connection of abscess foci between bilateral submandibular and submental area are observed on axial sections. d) Completely regressed abscesses are shown in the reformatted, contrast-enhanced CT images on the axial plane after the treatment.

multiloculated, large-sized and extended abscesses like the one in this study, the external approach is preferred (1). In this way, all loculations of the abscess can be drained, and the major vascular structures such as carotid arteries and internal jugular veins can be controlled (1).

In microbiological examination of parapharyngeal abscess material, both polymicrobial growth cases and cases where there is no growth can be observed. The most commonly isolated agents are Streptococcal species, Peptostreptococcus species, Staphylococcus aerius, Bacteroides fragilis, Fusobacterium necrophorum and Pseudomonas aeruginosa, but many other bacterial species can also be encountered (2-
4). Therefore, the treatment should be started empirically with aggressive and broad spectrum parenteral antibiotic therapy and should be re-evaluated according to the results of the antibiogram $(5,8)$. In line with the literature, non-hemolytic streptococcus, a streptococcal type bacterium, was isolated in our case. Treatment was continued since the agent isolated in the culture was sensitive to the empirically started treatment.

A parapharyngeal abscess can cause massive hemorrhages which can lead to carotid pseudoaneurysm or mycotic aneurysm. Cicek et al. managed to treat such a massive bleeding using the endovascular approach (9). In our case, massive bleedings were observed on the right and left side 
at one day intervals. However, it was revealed that these hemorrhages did not originate from the internal jugular vein or carotid artery, but from both the branches of the internal maxillary artery and the ascending pharyngeal artery. The occurrence of these hemorrhages despite the good bleeding control during the first surgery confirmed that the infection agents had eroded the vascular wall. We were able to control the bleedingwith the help of bipolar cauterization and surgicel ${ }^{\circledR}$ (Absorbable Hemostat, Ethicon Inc., Johnson \& Johnson, NJ 08933, USA).

Providing airway control is crucial in deep neck infections. For this reason, intubation and even tracheotomy can be applied when necessary $(2,4)$. Since opening the mouth could not be ensured in our case due to trismus, intubation could not be carried out during sudden respiratory distress. Because the neck structures were very rigid and had edema, tracheotomy was tried in the first place. However, due to the need to act quickly, a coniotomy was performed using the same incision. After the coniotomy, a tracheotomy was started in the same session and the abscess was drained on both sides.

In the case of respiratory arrest, asphyxia and death are inevitable unless steps are taken quickly. Indeed, in an autopsy case which was presented by Osculati and Fassina (10) about a 65 -year-old woman who died suddenly due to asphyxia, it was revealed that the cause of the mortality was left parapharyngeal abscess and concomitant subtotal closure of aditus laryngis. In our case, the bilateral nature of the parapharyngeal abscess, its extension into the sublingual and submandibular distances and its blocking of the air passage explained why the patient suddenly went into respiratory arrest. Although a caries was detected in the right molar tooth in our case, the question of why the abscess was bilateral needed to be answered. Contrary to what was expected, no spread was observed from the retropharyngeal to the parapharyngeal area in our case (Figure $2 \mathrm{~b}, \mathrm{c}$ ). This condition could have been caused by the fact that the infection extended from the submandibular area to the left parapharyngeal distance or could have been a result of a localized infection such as a gingivitis on the left side which regressed with the treatment. Continuation of abscess foci from the two submandibular areas to the submental area in axial CT sections strengthened the argument that bilateral parapharyngeal involvement occurred through the submental and submandibular pathway (Figure $2 b, c)$.

\section{CONCLUSION}

Bilateral parapharyngeal abscess is a very rare condition. Placing pressure on the airway on both sides increases the threat to respiratory safety. Moreover, when it is presented with trismus, the patient cannot be intubated. Here, the physician should be prepared for an emergency tracheotomy or coniotomy. In cases where the infection erodes the vascular wall, carotid artery bleeding should be considered in the first place, as typically indicated in the literature. However, it is important to keep in mind that external carotid artery branches such as the internal maxillary artery and ascending pharyngeal artery can also cause serious bleeding.

Informed Consent: Verbal informed consent was obtained from the patient who agreed to take part in the study.

Peer-Review: Externally peer-reviewed.

Author Contributions: Conception/Design of Study- I.E.; Drafting Manuscript- I.E., C.A.; Critical Revision of Manuscript- C.A.; Final Approval and Accountability- I.E., C.A., E.S., E.G.

Conflict of Interest: Authors declared no conflict of interest.

Financial Disclosure: Authors declared no financial support.

\section{REFERENCES}

1. Amar YG, Manoukian JJ. Intraoral drainage: Recommended as the initial approach for the treatment of parapharyngeal abscesses. Otolaryngol Head Neck Surg 2004;130(6):676-80.

2. Oh JH, Kim Y, Kim CH. Parapharyngeal Abscess: Comprehensive Management Protocol ORL 2007;69(1):37-42.

3. Alaani A, Griffiths H, Minhas SS, Olliff J, Lee ABD. Parapharyngeal abscess: diagnosis, complications and management in adults. Eur Arch Otorhinolaryngol 2005;262(4):345-50.

4. Klug TE, Fischer ASL, Antonsen C, Rusan M, Eskildsen H, Ovesen T. Parapharyngeal abscess is frequently associated with concomitant peritonsillar abscess. Eur Arch Otorhinolaryngol 2014;271(6):170107.

5. Page C, Biet A, Zaatar R, Strunski V. Parapharyngeal abscess: diagnosis and treatment. Eur Arch Otorhinolaryngol 2008;265(6):681-6.

6. Moiseenko AP. Bilateral parapharyngeal abscess complicated by sepsis and pulmonary candidiasis. Vestn Otorinolaringol 1962;24:91-2.

7. Lee $\mathrm{CH}$, Lee TJ, Chen CW. Transnasal endoscopic approach for drainage of pediatric parapharyngeal space abscess. Otolaryngol Head Neck Surg 2010;143(3):467-8.

8. Koivunen $\mathrm{P}$, Löppönen $\mathrm{H}$. Internal carotid artery thrombosis and Horner's syndrome as complications of parapharyngeal abscess. Otolaryngol Head Neck Surg 1999;121(1):160-2.

9. Cicek MT, Yildirim IO, Gunduz E. Endovascular Treatment of Carotid Pseudoaneurysm Bleeding Due to Parapharyngeal Abscess. J Craniofac Surg 2020;31(4):324-6.

10. Osculati A, Fassina G. Parapharyngeal abscess and sudden death. Legal Med 1999;1(1):34-6. 